\title{
BMJ Open Germline mutations in PMS2 and MLH1 in individuals with solitary loss of PMS2 expression in colorectal carcinomas from the Colon Cancer Family Registry Cohort
}

Christophe Rosty, ${ }^{1,2,3}$ Mark Clendenning, ${ }^{3}$ Michael D Walsh, ${ }^{4}$ Stine V Eriksen, ${ }^{3}$ Melissa C Southey, ${ }^{3}$ Ingrid M Winship, ${ }^{5,6}$ Finlay A Macrae, ${ }^{7}$ Alex Boussioutas, ${ }^{8,9,10}$ Nicola K Poplawski, ${ }^{11,12}$ Susan Parry, ${ }^{13,14}$ Julie Arnold, ${ }^{14}$ Joanne P Young, ${ }^{15,16,17}$ Graham Casey, ${ }^{18}$ Robert W Haile, ${ }^{19}$ Steven Gallinger, ${ }^{20}$ Loïc Le Marchand, ${ }^{21}$ Polly A Newcomb, ${ }^{22,23}$ John D Potter, ${ }^{22,23,24}$ Melissa DeRycke, ${ }^{25}$ Noralane M Lindor, ${ }^{26}$ Stephen N Thibodeau, ${ }^{27}$ John A Baron, ${ }^{28}$ Aung Ko Win, ${ }^{29}$ John L Hopper, ${ }^{29}$ Mark A Jenkins, ${ }^{29}$ Daniel D Buchanan, ${ }^{3,29}$ on behalf of the Colon Cancer Family Registry Cohort

To cite: Rosty C,

Clendenning M, Walsh MD, et al. Germline mutations in PMS2 and MLH1 in individuals with solitary loss of PMS2 expression in colorectal carcinomas from the Colon Cancer Family Registry Cohort. BMJ Open 2016;6:e010293. doi:10.1136/bmjopen-2015010293

- Prepublication history and additional material is available. To view please visit the journal (http://dx.doi.org/ 10.1136/bmjopen-2015010293).

Received 20 October 2015 Revised 12 January 2016 Accepted 29 January 2016

CrossMark

For numbered affiliations see end of article.

Correspondence to Dr Christophe Rosty; c.rosty@uq.edu.au

\section{ABSTRACT}

Objectives: Immunohistochemistry for DNA mismatch repair proteins is used to screen for Lynch syndrome in individuals with colorectal carcinoma (CRC). Although solitary loss of PMS2 expression is indicative of carrying a germline mutation in PMS2, previous studies reported MLH1 mutation in some cases. We determined the prevalence of $M L H 1$ germline mutations in a large cohort of individuals with a CRC demonstrating solitary loss of PMS2 expression.

Design: This cohort study included 88 individuals affected with a PMS2-deficient CRC from the Colon Cancer Family Registry Cohort. Germline PMS2 mutation analysis (long-range PCR and multiplex ligation-dependent probe amplification) was followed by MLH1 mutation testing (Sanger sequencing and multiplex ligation-dependent probe amplification).

Results: Of the 66 individuals with complete mutation screening, we identified a pathogenic PMS2 mutation in $49(74 \%)$, a pathogenic MLH1 mutation in $8(12 \%)$ and a $M L H 1$ variant of uncertain clinical significance predicted to be damaging by in silico analysis in 3 $(4 \%) ; 6(9 \%)$ carried variants likely to have no clinical significance. Missense point mutations accounted for most alterations $(83 \% ; 9 / 11)$ in $M L H 1$. The $M L H 1$ c. $113 \mathrm{~A}>\mathrm{G}$ p.Asn38Ser mutation was found in 2 related individuals. One individual who carried the $M L H 1$ intronic mutation c. $677+3 \mathrm{~A}>\mathrm{G}$ p.G $\ln 197 \mathrm{Argfs}{ }^{\star} 8$ leading to the skipping of exon 8 , developed 2 tumours, both of which retained MLH1 expression.

Conclusions: A substantial proportion of CRCs with solitary loss of PMS2 expression are associated with a deleterious $M L H 1$ germline mutation supporting the screening for MLH1 in individuals with tumours of this immunophenotype, when no PMS2 mutation has been identified.

\section{Strengths and limitations of this study}

- Largest reported sample of colorectal cancers with solitary loss of PMS2 expression.

- Most comprehensive approaches used for testing for germline PMS2 mutations.

- Multicentre setting which may affect the consistency in the formalin fixation conditions of tissue blocks and lead to immunostaining artefacts.

- No mutation screening data available for 20 cases $(24 \%)$.

- Selected cases (young individuals with strong family history of colorectal carcinoma (CRC)) that may not reflect the actual rate of PMS2-deficient CRC in the general population and the mutation rates in PMS2 and MLH1.

\section{INTRODUCTION}

Lynch syndrome is an autosomal-dominant inherited condition defined by the identification of a germline mutation in a DNA mismatch repair (MMR) gene $(M L H 1, M S H 2$, PMS2 or MSH6), or in the EPCAM gene, leading to constitutional epigenetic silencing of the downstream $\mathrm{MSH} 2$ gene. $^{1}$ Individuals who carry a MMR gene mutation are at an increased risk of developing cancers at multiple sites, most notably colorectal and endometrial carcinomas, but also carcinomas from the upper urinary tract, pancreas, hepatobiliary tract, stomach, small intestine and ovaries. $^{2}$

The current diagnostic approach for the identification of individuals with an MMR 
gene mutation is a multistep process in which pathologists play an instrumental role. Tumours arising in individuals with an MMR gene mutation demonstrate high levels of microsatellite instability (MSI) secondary to altered DNA MMR mechanisms in tumour cells. Immunohistochemistry for DNA MMR proteins is widely used to identify MMR deficiency in colorectal carcinomas (CRCs) as a screen for MMR gene carriers. ${ }^{3}$ Of all abnormal patterns of immunohistochemical results, loss of expression of MLH1 and PMS2 is the most common. MLH1 and PMS2 function as a stable heterodimer that, along with MSH2, MSH6 and EXO1, corrects small errors involving mispaired nucleotides which are introduced by DNA polymerase during DNA replication. A functional defect in $M L H 1$ results in the degradation of both MLH1 and PMS2, whereas a defect in PMS2 results only in the degradation of PMS2. Consequently, loss of expression of MLH1 and PMS2 in CRC generally indicates an alteration in $M L H 1$, either by somatic methylation of the $M L H 1$ promoter region (sporadic cases) or by a $M L H 1$ germline mutation (Lynch syndrome), and solitary loss of PMS2 expression generally indicates an underlying germline defect in PMS2.

Inconsistent immunohistochemical results have been reported, in particular the retained expression of MLH1 in tumours from individuals with a germline $M L H 1$ mutation. ${ }^{4-8}$ This phenomenon can be misleading if PMS2 immunostaining is not performed. We sought to confirm that germline mutations in MLH1 may underlie a substantial proportion of CRC with solitary loss of PMS2 expression. To address this question, we performed mutation analysis of the MLH1 and PMS2 genes in individuals from the Colon Cancer Family Registry Cohort whose tumours showed solitary loss of PMS2.

\section{MATERIALS AND METHODS}

\section{Study participants}

Participants were probands and relatives from families recruited between 1997 and 2012 to the Colon Cancer Family Registry Cohort via both population-based recruitment and clinic-based recruitment in Australasia and North America. ${ }^{9}$ All CRC cases were reviewed by specialist gastrointestinal pathologists for histological type and grade. ${ }^{10}$ Tumours from the caecum, ascending colon, hepatic flexure and transverse colon were considered proximal tumours. Immunohistochemistry for DNA MMR proteins MLH1, PMS2, MSH2 and MSH6 was performed as previously described. ${ }^{3}$ A subset of tumours were analysed for MSI status from formalin-fixed paraffin-embedded tissue as previously described. ${ }^{3}$ Individuals were eligible for this study if they had a histologically confirmed diagnosis of CRC with an immunohistochemical profile of the DNA MMR proteins, demonstrating presence of expression of the MLH1 protein and concomitant loss of expression of the PMS2 protein. The somatic $\mathrm{T}>\mathrm{A}$ mutation at nucleotide 1799 in exon 15 of the $B R A F$ gene (BRAF ${ }^{V 600 \mathrm{E}}$ mutation) was detected using fluorescent allele-specific PCR. ${ }^{11}$ MLH1 promoter methylation was analysed using the MLH1-M2 methylight reaction using an Arthobacter luteus (ALU) control reaction to normalise for bisulfite-converted input DNA. ${ }^{12}$ Informed consent was obtained from all participants to collect a blood sample and tumour pathology materials (tumour blocks and slides). Ethics approval was obtained from the relevant institutional Human Research Ethics Committees at recruiting centres.

\section{Family history of cancer}

Information on personal and family history of CRC and other cancers in first-degreeand second-degree relatives was obtained via standardised questionnaires at the time of baseline recruitment. Cancer diagnoses were verified, where possible, using pathology reports, medical records, cancer registry reports and death certificates. Probands and relatives were either actively or passively followed up approximately every 5 years from baseline enrolment, including the collection of updated information by linkage to tumour registries and death indices on the number, sex and birthdates of first-degree relatives, their cancer history, vital status and, if deceased, date of death. All cancers, except for non-melanoma skin cancers, were recorded with dates of diagnosis. The present study was based on all available baseline and follow-up data. Family history of cancer that fulfilled either the Amsterdam I or II criteria were determined. ${ }^{13}$

\section{Germline mutation testing}

Germline mutation testing for the individuals in this study primarily involved testing for $P M S 2$ gene mutations and when a PMS2 mutation was not identified, germline mutation testing of the MLH1 gene was conducted. PMS2 was screened for germline mutations using a DNA-based, best practice, approach combining longrange PCR and multiplex ligation-dependent probe amplification (MLPA). Briefly, for point mutation analysis, parts of the PMS2 gene (exons 1-5, 9 and 11-15) were specifically targeted, while avoiding pseudogene sequences, via a set of three long-range PCRs (TaKaRa LA Taq; TaKaRa Bio Inc, Shiga, Japan). These longrange products are then used as the template for a set of PMS2-specific exonic PCRs (see online supplementary table S1 for primer sequences). To assess for large-scale (whole exon) deletions, we used the P008-B1 MLPA kit according to the manufacturer's instructions (MRC-Holland; Amsterdam, The Netherlands). To accurately call PMS2 mutations at the $3^{\prime}$ end of the gene, the MLPA kit contains probes targeted to paralogous sequence variants which requires pseudogene-specific sequence data to interpret the findings. Pseudogene sequences were obtained as above, replacing the PMS2-specific long-range amplicon with a pseudogene-specific amplicon. Germline mutation testing for $M L H 1$ was performed by Sanger sequencing as previously described. ${ }^{9}{ }^{14}$ Large duplication and deletion mutations were detected by MLPA. Germline 
variants within the MLH1 and PMS2 genes were classified for pathogenicity based on the InSiGHT database classifications $^{15} \quad$ (http://insight-group.org/variants/ classifications/). If no classification was available, the predicting effect of an unclassified variant (UV) to the protein function was assessed in silico using the 'Sorting Tolerant From Intolerant' (SIFT) and the 'Polymorphism Phenotyping v2' (PolyPhen-2) web-based algorithms. ${ }^{1617}$

\section{Statistical analysis}

Statistical analyses were performed with SPSS statistics software V.17.0 (SPSS Inc, Chicago, Illinois, USA). Comparisons for categorical variables were performed using Pearson's $\chi^{2}$ test or Fisher's exact test where appropriate. Student t test was used for continuous variables. A two-tailed $\mathrm{p}$ value was used for all analyses and values less than 0.05 were considered to be significant.

\section{RESULTS}

The study included 90 CRCs from 88 individuals demonstrating loss of PMS2 expression and normal retained MLH1 expression by immunohistochemistry. They had a mean age at CRC diagnosis of $51.7_{ \pm}$SD 12.4 years and included $57 \%$ males. MSI status was available for $46 / 90$ CRCs (51\%), with high levels of MSI observed in 42/46 $(91 \%)$ cases. $M L H 1$ methylation and/or a $B R A F^{V 600 E}$ mutation were present in 4 of the 90 CRCs that were excluded from the study. Six CRCs $(7 \%)$ also showed loss of MSH6 protein expression. Four individuals were not tested for PMS2 and MLH1 mutations due to the unavailability of blood-derived DNA, and complete gene testing was not possible for a further 14 individuals (figure 1). The final study group consisted of 66 individuals with complete screening for germline mutations in the PMS2 and MLH1 genes. A pathogenic PMS2 germline mutation was identified in 49 individuals (74\%; see online supplementary table S2), some of which were reported previously. ${ }^{18}$ Variants in the MLH1 gene were identified in 11 individuals (17\%). In eight individuals, the variants were classified as pathogenic mutations (class 5); in the other three individuals, variants were unclassified but predicted to be damaging by SIFT and PolyPhen-2 algorithms (table 1 and figure 2).

Immunostained slides were reviewed in 5 of these 11 cases, confirming the retained expression of MLH1 and the loss of PMS2 expression in carcinoma cells. No mutation within PMS2 or MLH1 could be found in the remaining six individuals $(9 \%)$. The clinicopathological characteristics of the PMS2 mutation carriers, the MLH1 pathogenic mutation and UV carriers and those individuals tested but found not to have a mutation in PMS2 or $M L H 1$ are shown in table 2.

The mean age at CRC diagnosis of the individuals with a MLH1 mutation or UV was significantly younger than those individuals with a PMS2 mutation $(\mathrm{p}=0.046)$. Amsterdam criteria I or II were less frequently found in
PMS2 mutation carriers compared with $M L H 1$ variant carriers $(\mathrm{p}=0.001)$.

Missense variants were the most common $M L H 1$ alteration identified, in eight individuals $(83 \%)$. The $M L H 1$ c.113A $>$ G p.Asn38Ser variant was found in two related individuals (cases 2 and 3). One individual who carried the intronic $M L H 1$ germline mutation c. $677+3 \mathrm{~A}>\mathrm{G}$ p. Gln197Argfs*8, which leads to the skipping of exon 8, developed two CRCs both of which retained MLH1 expression (cases 5 and 6). One individual carried a splice site mutation leading to an in-frame deletion of two exons (case 4) and one individual carried a small insertion resulting in a frameshift mutation (case 7; table 1).

\section{DISCUSSION}

To assess the possible role of MLH1 mutations in CRCs showing solitary loss of PMS2 expression by immunohistochemistry, we studied a series of 90 CRCs from 88 individuals from the Colon Cancer Family Registry Cohort with this immunophenotype. Among the 66 individuals with complete germline mutation analysis, we identified a pathogenic PMS2 mutation in 49 cases $(74 \%)$ and a pathogenic $M L H 1$ mutation in 8 cases (12\%). A further three cases $(4 \%)$ had a variant of uncertain clinical significance in $M L H 1$ predicted to be damaging, and six cases $(9 \%)$ had no identifiable variant likely to have clinical significance in either gene. Moreover, a high proportion of the MLH1 variants identified resulted in missense changes, suggesting that a non-functional MLH1 protein that retains its MLH1 antigenicity is a conceivable explanation.

Immunohistochemistry for the DNA MMR proteins MLH1, PMS2, MSH2 and MSH6 in CRC is a highly sensitive test to screen for Lynch syndrome, with 93-100\% concordance with MSI testing. ${ }^{3}{ }^{4}$ However, false-negative results for MLH1 immunohistochemistry have been reported in small series. In a study evaluating the benefit of adding PMS2 to MLH1 staining, de Jong et at found eight MLH1 mutations (42\%) compared with only three PMS2 mutations (16\%) out of 19 CRCs demonstrating solitary loss of PMS2 expression. When considering all the MLH1 mutations identified in their study, a high proportion $(8 / 35 ; 23 \%)$ showed loss only of PMS2 expression while retaining expression of MLH1. A large deletion of exons 14-19 of MLH1 was also reported in 2 of $8(25 \%)$ CRC with solitary PMS2 loss of expression in a separate study. ${ }^{5}$ A recent study of 16 CRCs and 16 endometrial carcinomas from 31 individuals, all with solitary loss of PMS2 expression, explored the frequency of MLH1 mutations in this group. ${ }^{19}$ Of the 17 individuals who subsequently had germline mutation testing of the MLH1 and PMS2 genes, six had pathogenic mutations in PMS2 (35\%), two had variants of uncertain clinical significance in PMS2 (12\%), four had MLH1 pathogenic mutations (24\%) whereas five had no mutation identified in either gene (29\%). 
Figure 1 Flow diagram of the study. CRC, colorectal carcinoma.

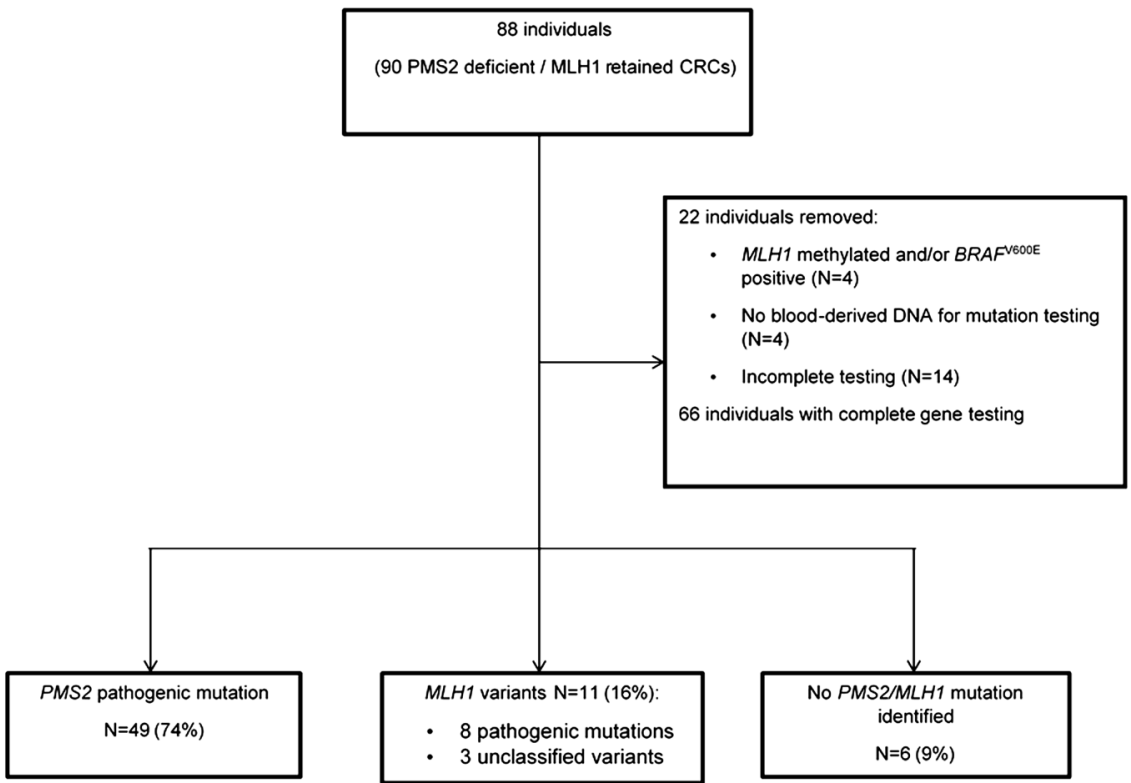

When restricted to patients with a CRC, a deleterious germline mutation in $M L H 1$ was reported in two of nine tested patients $(22 \%)$. Compared with these studies, our rate of $P M S 2$ mutation in 66 tested individuals was higher at $74 \%$ and the rate of $M L H 1$ deleterious mutation slightly lower at $12 \%$. Two cousins (tumours 2 and 3) who carried the same MLH1 mutation both had CRC with solitary PMS2 loss. Similarly, one individual, who carried the MLH1 c. $677+3 \mathrm{~A}>\mathrm{G}$ p.Gln197Argfs*8 mutation, developed two CRCs with solitary PMS2 loss. Both these examples suggest that it is the nature of the mutation rather than a technical anomaly associated with tissue fixation or staining quality that is the cause of this differential staining pattern. In support of this, Zighelboim et $a l^{20}$ described two sisters who carried the same MLH1 mutation: one developed endometrial cancer at 48 years and the other CRC at 45 years and endometrial cancer at 53 years; all tumours showed solitary loss of PMS2 expression and the presence of MLH1 expression.

A trend towards universal CRC tumour immunohistochemistry will increase the detection of abnormal staining patterns that require interpretation. This allows the most probable cause to be decided and thus the most appropriate management instituted. A solitary loss of PMS2 expression is suggestive of Lynch syndrome with a primary defect in the PMS 2 gene. Interestingly, we identified MLH1 methylation or the somatic BRAF $F^{V 600 E}$ mutation in four cases, indicating that isolated PMS2 loss of expression can occur outside Lynch syndrome. It may

Table 1 Characteristics of the 11 individuals with a germline MLH1 variant from 12 colorectal carcinomas with loss of PMS2 expression and retained MLH1 expression

\begin{tabular}{|c|c|c|c|c|c|c|c|}
\hline $\begin{array}{l}\text { Tumour } \\
\#\end{array}$ & Gender & $\begin{array}{l}\text { Age, } \\
\text { years }\end{array}$ & $\begin{array}{l}\text { Amsterdam } \\
\text { criteria }\end{array}$ & $\begin{array}{l}\text { Tumour } \\
\text { location }\end{array}$ & Variant & Protein & $\begin{array}{l}\text { InSiGHT } \\
\text { classification }\end{array}$ \\
\hline 1 & Female & 40 & None & Descending & c. $230 \mathrm{G}>\mathrm{A}$ & p.Cys77Tyr & Class 5 \\
\hline $2^{*}$ & Male & 44 & None & Descending & c. $113 \mathrm{~A}>\mathrm{G}$ & p.Asn38Ser & Class 5 \\
\hline $3^{*}$ & Male & 40 & I & Rectum & c. $113 A>G$ & p.Asn38Ser & Class 5 \\
\hline 4 & Female & 51 & 1 & Descending & c. $790+1 \mathrm{G}>\mathrm{A}$ & p.Glu227_Ser295del & Class 5 \\
\hline $5 \dagger$ & Male & 34 & II & Cecum & c. $677+3 A>G$ & p.GIn197Argfs ${ }^{\star} 8$ & Class 5 \\
\hline $6 \dagger$ & Male & 34 & II & Rectum & c. $677+3 A>G$ & p.GIn197Argfs*8 & Class 5 \\
\hline 7 & Male & 63 & 1 & Caecum & c.2195_2198dup & p.His733Glnfs*14 & Class 5 \\
\hline 8 & Male & 49 & None & Unknown & c. $230 \mathrm{G}>\mathrm{A}$ & p.Cys77Tyr & Class 5 \\
\hline 9 & Female & 33 & None & Rectum & c. $199 \mathrm{G}>\mathrm{A}$ & p.Gly67Arg & Class 5 \\
\hline 10 & Male & 62 & II & Transverse & c. $374 \mathrm{C}>\mathrm{A}$ & p.Ala125Glu & UV \\
\hline 11 & Male & 24 & None & Ascending & c. $187 \mathrm{G}>\mathrm{C}$ & p.Asp63His & UV \\
\hline 12 & Male & 38 & I & Cecum & c. $187 \mathrm{G}>\mathrm{C}$ & p.Asp63His & UV \\
\hline
\end{tabular}

UV: unclassified variant by InSiGHT. These UVs were predicted to be damaging through in silico analysis.

${ }^{*}$ Cousins.

†2xcolorectal carcinomas from the same individual. 
Figure 2 Graphical overview of the location of the 11 MLH1 mutations identified. Numbers above the gene schematic denote the amount of mutations identified in the corresponding exons.

Mutation subtypes are boxed in green and the predicted functional domains of the MLH1 protein are displayed below the gene schematic.

therefore be useful to test PMS2-deficient CRC for $B R A F^{V 600 E}$ mutation or $M L H 1$ methylation to exclude sporadic tumour. Screening for PMS2 mutations has been problematic due a large number of homologous sequences within pseudogenes that closely flank the functional gene and most likely accounts for the lower proportion of $P M S 2$ mutations reported in previous studies. The recent development of new methods incorporating long-range PCR and MLPA has eliminated most of the previous problems, such that the identification of large-scale deletions of exons 3 and/or 4 are now the only difficulty. The results from this study, representing the largest number of CRC with solitary loss of PMS2, support germline mutation screening of $M L H 1$ when no mutation in PMS2 has been found. However, a substantial proportion of MMR-deficient CRCs with no evidence of $M L H 1$ methylation or $B R A F^{V 600 E}$ mutation remain unexplained and are referred to as Lynch-like or suspected Lynch syndrome. A number of potential causes for the underlying loss of PMS2 protein expression in these cases, including biallelic somatic mutations and cryptic mutations, have been described in a recent review. ${ }^{21}$ In a large population-based study of the Colon Cancer Family Registry Cohort, 5.6\% (271/4 853) of all CRCs were classified as Lynch-like syndrome, representing $56 \%$ of all MMR-deficient CRCs not secondary to
MLH1 methylation. In our study, six CRCs showed concurrent loss of MSH6 and PMS2. The most likely explanation for the loss of MSH6 expression in these six cases is the somatic frameshift mutation in the $(\mathrm{C}) 8$ microsatellite in exon 5 of the MSH6 gene secondary to the loss of MMR function resulting from the PMS2 defect. ${ }^{22}$ The use of panel testing rather than a single-gene approach would be useful; this is of particular interest clinically, where the PMS2 gene has lower penetrance than other MMR genes ${ }^{23}$ and family history is a suboptimal way of finding potentially high-risk families, where risk assessment and risk management has improved outcomes. However, PMS2 testing remains challenging even by next generation sequencing due to its complex structure.

Our study included the largest reported sample of CRCs with solitary loss of PMS2 to date. Testing for germline PMS2 mutations used in this study employed the most up-to-date and comprehensive approaches described, ${ }^{18} 24$ as demonstrated by the high rate of identified PMS2 mutations. One limitation of this study is the multicentre setting which may affect the consistency in the formalin fixation conditions of tissue blocks and lead to immunostaining artefacts. Other limitations include the absence of other Lynch syndrome-associated tumours, and the lack of mutation screening data for 20 $(24 \%)$ cases. Moreover, our results may not reflect the

Table 2 Characteristics of 66 individuals with 68 colorectal carcinomas (CRCs) showing loss of PMS2 expression and retained MLH1 expression

\begin{tabular}{|c|c|c|c|c|c|}
\hline Group & $\begin{array}{l}\text { All (68 CRCs } \\
\text { from } 66 \\
\text { individuals) } \\
\end{array}$ & $\begin{array}{l}\text { PMS2 mutations } \\
\text { ( } N=50 \text { CRCs from } \\
49 \text { individuals) }\end{array}$ & $\begin{array}{l}\text { MLH1 variant } \\
\text { ( } \mathrm{N}=12 \text { CRCs from } \\
11 \text { individuals) }\end{array}$ & $\begin{array}{l}\text { P value PMS2 } \\
\text { mutations vs } \\
M L H 1 \text { variant }\end{array}$ & $\begin{array}{l}\text { No PMS2 or } \\
\text { MLH1 } \\
\text { mutation } \\
(\mathrm{N}=6)\end{array}$ \\
\hline $\begin{array}{l}\text { Mean age at diagnosis } \pm \text { SD } \\
\text { (range) in years }\end{array}$ & $\begin{array}{l}51.7 \pm 11.7 \\
(24-80)\end{array}$ & $\begin{array}{l}52.1 \pm 11.3 \\
(35-80)\end{array}$ & $\begin{array}{l}43.5 \pm 12.0 \\
(24-63)\end{array}$ & 0.046 & $\begin{array}{l}52.2 \pm 11.7 \\
(33-69)\end{array}$ \\
\hline Gender male, N (\%) & $36(54.5)$ & $26 / 49(53.1)$ & $8 / 11(72.7)$ & 0.32 & $2(33.3)$ \\
\hline Amsterdam criteria I, N (\%) & $6(9.1)$ & $0(0)$ & $6(54.5)$ & $0.001^{*}$ & $0(0)$ \\
\hline Amsterdam criteria II, N (\%) & $10(15.2)$ & $4(8.2)$ & $6(54.5)$ & & $0(0)$ \\
\hline Proximal CRC location, N (\%) & $42 / 64(65.6)$ & $35 / 47(74.5)$ & $5 / 10(50)$ & 0.14 & $2 / 6(33.3)$ \\
\hline Histological type, N (\%) & & & & 1 & \\
\hline Adenocarcinoma & $50 / 63(79.4)$ & $36 / 47(76.6)$ & 9/11 (81.8) & & $6 / 6(100)$ \\
\hline Mucinous carcinoma & $13 / 63(20.6)$ & $11 / 47(23.4)$ & 2/11 (18.2) & & $0 / 6(0)$ \\
\hline Histological grade, N (\%) & & & & 0.024 & \\
\hline Well/moderate & $42 / 62(67.7)$ & $36 / 46(78.3)$ & $4 / 10(40)$ & & $1 / 5(20)$ \\
\hline Poor & 20/62 (32.3) & $10 / 46(21.7)$ & $6 / 10(60)$ & & $4 / 5(80)$ \\
\hline
\end{tabular}


actual rate of PMS2-deficient CRC in the general population and the mutation rates in PMS2 and MLH1, as these cases were selected in young individuals with strong family history of CRC.

In conclusion, the findings from this study suggest that CRCs in MLH1 mutation carriers can demonstrate a normal pattern of MLH1 expression and justify the testing for $M L H 1$ germline mutation in individuals with a CRC showing solitary loss of PMS2 expression when a PMS2 mutation is not identified.

\section{Author affiliations}

${ }^{1}$ Envoi Pathology, Brisbane, Queensland, Australia

${ }^{2}$ The School of Medicine, The University of Queensland, Brisbane, Queensland, Australia

${ }^{3}$ Genetic Epidemiology Laboratory, Department of Pathology, The University of Melbourne, Parkville, Victoria, Australia

${ }^{4}$ Department of Histopathology, Sullivan Nicolaides Pathology, Brisbane, Queensland, Australia

${ }^{5}$ Department of Medicine, The University of Melbourne, Parkville, Victoria, Australia

${ }^{6}$ Genetic Medicine and Family Cancer Clinic, Royal Melbourne Hospital, Parkville, Victoria, Australia

${ }^{7}$ Colorectal Medicine and Genetics, The Royal Melbourne Hospital, Parkville, Victoria, Australia

${ }^{8}$ Department of Medicine, Royal Melbourne Hospital, The University of Melbourne, Parkville, Victoria, Australia

${ }^{9}$ Cancer Genomics and Predictive Medicine, Peter MacCallum Cancer Centre, East Melbourne, Victoria, Australia

${ }^{10}$ Sir Peter MacCallum Department of Oncology, The University of Melbourne, Parkville, Victoria, Australia

${ }^{11}$ South Australian Clinical Genetics Service, SA Pathology at the WCH, North Adelaide, South Australia, Australia

${ }^{12}$ University Department of Paediatrics, University of Adelaide, Adelaide, South Australia, Australia

${ }^{13}$ New Zealand Familial Gastrointestinal Cancer Registry, Auckland City

Hospital, Auckland, New Zealand

${ }^{14}$ Department of Gastroenterology, Middlemore Hospital, Auckland, New Zealand

${ }^{15}$ Department of Haematology and Oncology, The Queen Elizabeth Hospital, Woodville, South Australia, Australia

${ }^{16}$ School of Medicine, University of Adelaide, Adelaide, South Australia, Australia

${ }^{17}$ SAHMRI Colorectal Node, Basil Hetzel Institute for Translational Research, Woodville, South Australia, Australia

${ }^{18}$ Department of Preventive Medicine, Keck School of Medicine and Norris Comprehensive Cancer Center, University of Southern California, Los Angeles, California, USA

${ }^{19}$ Department of Medicine, Division of Oncology, Stanford Cancer Institute, Stanford University, Stanford, California, USA

${ }^{20}$ Lunenfeld Tanenbaum Research Institute, Mount Sinai Hospital, University of Toronto, Toronto, Ontario, Canada

${ }^{21}$ University of Hawaii Cancer Center, Honolulu, Hawaii, USA

${ }^{22}$ Public Health Sciences Division, Fred Hutchinson Cancer Research Center, Seattle, Washington, USA

${ }^{23}$ School of Public Health, University of Washington, Seattle, Washington, USA

${ }^{24}$ Centre for Public Health Research, Massey University, Wellington, New Zealand

${ }^{25}$ Departments of Health Sciences Research, Biomedical Statistics and Informatics, Laboratory Medicine and Pathology, Medical Genetics, Medical Genomics Technology and Advanced Genomics Technology Center, Mayo Clinic College of Medicine, Rochester, Minnesota, USA

${ }^{26}$ Department of Health Science Research, Mayo Clinic Arizona, Scottsdale, Arizona, USA

${ }^{27}$ Molecular Genetics Laboratory, Department of Laboratory Medicine and Pathology, Mayo Clinic, Rochester, Minnesota, USA
${ }^{28}$ Department of Medicine, University of North Carolina, Chapel Hill, North Carolina, USA

${ }^{29}$ Centre for Epidemiology and Biostatistics, Melbourne School of Population and Global Health, The University of Melbourne, Parkville, Victoria, Australia

Acknowledgements The authors thank all study participants of the Colon Cancer Family Registry and staff for their contributions to this project, in particular Sue Preston, Allyson Templeton, Teresa Selander and Maggie Angelakos.

Contributors $\mathrm{CR}, \mathrm{MC}$, DDB were involved in design of the study, acquisition, analysis and interpretation of data, drafting the manuscript and final approval of the version to be published. MDW, SVE, JPY were involved in acquisition, analysis and interpretation of data, revising the manuscript critically and final approval of the version to be published. MCS, IMW, FAM, AB, NKP, SP, JA, GC, RWH, SG, LLM, PAN, JDP, MD, NML, SNT, JAB, AKW, JLH, MAJ were involved in data acquisition, revising the manuscript critically and final approval of the version to be published.

Funding This work was supported by grant UM1 CA167551 from the National Cancer Institute and through cooperative agreements with the following Colon Cancer Family Registry centres: Australasian Colorectal Cancer Family Registry (U01 CA074778 and U01/U24 CA097735), Mayo Clinic Cooperative Family Registry for Colon Cancer Studies (U01/U24 CA074800), Ontario Familial Colorectal Cancer Registry (U01/U24 CA074783), Seattle Colorectal Cancer Family Registry (U01/U24 CA074794), University of Hawaii Colorectal Cancer Family Registry (U01/U24 CA074806), USC Consortium Colorectal Cancer Family Registry U01/U24 CA074799). AKW is an Australian National Health and Medical Research Council (NHMRC) Early Career Fellow. MCS is a NHMRC Senior Research Fellow. JLH is a NHMRC Senior Principal Research Fellow and Distinguished Visiting Professor at Seoul National University, Korea. MAJ is a NHMRC Senior Research Fellow. DDB is a University of Melbourne Research at Melbourne Accelerator Program (R@MAP) Senior Research Fellow. This project was conducted under Colon Cancer Family Registry approval C-CP-0309-01-A1.

Disclaimer The content of this manuscript does not necessarily reflect the views or policies of the National Cancer Institute or any of the collaborating centres in the CFRs, nor does mention of trade names, commercial products, or organisations imply endorsement by the US Government or the CFR. Authors had full responsibility for the design of the study, the collection of the data, the analysis and interpretation of the data, the decision to submit the manuscript for publication, and the writing of the manuscript.

Patient consent Obtained.

Ethics approval Multi-institutional Human Research Ethics Committees.

Provenance and peer review Not commissioned; externally peer reviewed.

Data sharing statement No additional data are available.

Open Access This is an Open Access article distributed in accordance with the Creative Commons Attribution Non Commercial (CC BY-NC 4.0) license, which permits others to distribute, remix, adapt, build upon this work noncommercially, and license their derivative works on different terms, provided the original work is properly cited and the use is non-commercial. See: http:// creativecommons.org/licenses/by-nc/4.0/

\section{REFERENCES}

1. Lynch PM. Hyperplastic polyposis: semantics, biology, and endoscopy. Gut 2010;59:1019-21.

2. Win AK, Young JP, Lindor NM, et al. Colorectal and other cancer risks for carriers and noncarriers from families with a DNA mismatch repair gene mutation: a prospective cohort study. $J$ Clin Oncol 2012;30:958-64.

3. Lindor NM, Burgart LJ, Leontovich O, et al. Immunohistochemistry versus microsatellite instability testing in phenotyping colorectal tumors. J Clin Oncol 2002;20:1043-8.

4. de Jong $A E$, van Puijenbroek $M$, Hendriks $Y$, et al. Microsatellite instability, immunohistochemistry, and additional PMS2 staining in suspected hereditary nonpolyposis colorectal cancer. Clin Cancer Res 2004;10:972-80. 
5. Halvarsson B, Lindblom A, Rambech E, et al. The added value of PMS2 immunostaining in the diagnosis of hereditary nonpolyposis colorectal cancer. Fam Cancer 2006;5:353-8.

6. van Riel E, Ausems MG, Hogervorst FB, et al. A novel pathogenic MLH1 missense mutation, c.112A > C, p.Asn38His, in six families with Lynch syndrome. Hered Cancer Clin Pr 2010;8:7.

7. Thibodeau SN, French AJ, Roche PC, et al. Altered expression of $\mathrm{hMSH} 2$ and $\mathrm{hMLH} 1$ in tumors with microsatellite instability and genetic alterations in mismatch repair genes. Cancer Res 1996;56:4836-40

8. Salahshor S, Koelble K, Rubio C, et al. Microsatellite instability and hMLH1 and hMSH2 expression analysis in familial and sporadic colorectal cancer. Lab Invest 2001;81:535-41.

9. Newcomb PA, Baron J, Cotterchio M, et al. Colon Cancer Family Registry: an international resource for studies of the genetic epidemiology of colon cancer. Cancer Epidemiol Biomark Prev 2007;16:2331-43.

10. Rosty C, Young JP, Walsh MD, et al. Colorectal carcinomas with KRAS mutation are associated with distinctive morphological and molecular features. Mod Pathol 2013;26:825-34.

11. Buchanan DD, Win AK, Walsh MD, et al. Family history of colorectal cancer in BRAF p.V600E-mutated colorectal cancer cases. Cancer Epidemiol Biomark Prev 2013;22:917-26.

12. Poynter JN, Siegmund KD, Weisenberger DJ, et al. Molecular characterization of MSI-H colorectal cancer by MLHI promoter methylation, immunohistochemistry, and mismatch repair germline mutation screening. Cancer Epidemiol Biomark Prev 2008;17:3208-15.

13. Vasen HF, Watson P, Mecklin JP, et al. New clinical criteria for hereditary nonpolyposis colorectal cancer (HNPCC, Lynch syndrome) proposed by the International Collaborative group on HNPCC. Gastroenterology 1999;116:1453-6.

14. Walsh MD, Buchanan DD, Cummings MC, et al. Lynch syndrome-associated breast cancers: clinicopathologic characteristics of a case series from the colon cancer family registry. Clin Cancer Res 2010;16:2214-24.
15. Thompson BA, Spurdle AB, Plazzer JP, et al. InSiGht. Application of a 5-tiered scheme for standardized classification of 2,360 unique mismatch repair gene variants in the InSiGHT locus-specific database. Nat Genet 2014;46:107-15.

16. Kumar P, Henikoff S, Ng PC. Predicting the effects of coding non-synonymous variants on protein function using the SIFT algorithm. Nat Protoc 2009;4:1073-81.

17. Adzhubei I, Jordan DM, Sunyaev SR. Predicting functional effect of human missense mutations using PolyPhen-2. Curr Protoc Hum Genet 2013;Chapter 7:Unit7.20.

18. Clendenning M, Macrae FA, Walsh MD, et al., Colorectal Cancer Family Registry. Absence of PMS2 mutations in colon-CFR participants whose colorectal cancers demonstrate unexplained loss of MLH1 expression. Clin Genet 2013;83:591-3.

19. Dudley B, Brand RE, Thull D, et al Germline MLH1 mutations are frequently identified in Lynch syndrome patients with colorectal and endometrial carcinoma demonstrating isolated loss of PMS2 immunohistochemical expression. Am J Surg Pathol 2015;39:1114-20.

20. Zighelboim I, Powell MA, Babb SA, et al. Epitope-positive truncating MLH1 mutation and loss of PMS2: implications for IHC-directed genetic testing for Lynch syndrome. Fam Cancer 2009;8:501-4.

21. Buchanan DD, Rosty $\mathrm{C}$, Clendenning $\mathrm{M}$, et al. Clinical problems of colorectal cancer and endometrial cancer cases with unknown cause of tumor mismatch repair deficiency (suspected Lynch syndrome). Appl Clin Genet 2014;7:183-93.

22. Shia J, Holck S, Depetris G, et al. Lynch syndrome-associated neoplasms: a discussion on histopathology and immunohistochemistry. Fam Cancer 2013;12:241-60.

23. Senter L, Clendenning M, Sotamaa K, et al. The clinical phenotype of Lynch syndrome due to germ-line PMS2 mutations. Gastroenterology 2008;135:419-28.

24. Vaughn CP, Baker CL, Samowitz WS, et al. The frequency of previously undetectable deletions involving 3' exons of the PMS2 gene. Genes Chromosomes Cancer 2013;52:107-12. 